\title{
Antimicrobial Pharmacokinetics and Pharmacodynamics in the Treatment of Nosocomial Gram-negative Infections
}

\author{
Raymond Cha ${ }^{1,2 *}$, Sarah M. Michienzi ${ }^{1}$ and Lama Hsaiky ${ }^{1,2}$ \\ ${ }^{1}$ Eugene Applebaum College of Pharmacy and Health Sciences, Wayne State University, 259 Mack Ave., Detroit, Michigan 48201, USA \\ 2Oakwood Healthcare System, 18101 Oakwood Blvd., Dearborn, Michigan 48124, USA
}

\begin{abstract}
While infections due to multidrug-resistant Gram-negative organisms overwhelm hospitals worldwide, preservation of current antimicrobial treatment options becomes paramount in the face of dwindling development of novel antibiotics. There is extensive data demonstrating that application of pharmacokinetic/pharmacodynamic principles improves the possibility of enhancing clinical and microbiological outcome. There is expanding evidence demonstrating that unfavorable clinical outcomes and antimicrobial resistance may be suppressed when specific pharmacokinetic/pharmacodynamic targets are attained for beta-lactam and carbapenem antibiotics. This paper highlights pertinent studies that contribute to the principles and application of these principles.
\end{abstract}

Keywords: Pharmacokinetics; Pharmacodynamics; Gram-negative; Resistance; Beta-lactam; Carbapenem; Continuous, Extended

\section{Introduction}

Multiple surveillance studies from across the globe report widespread multi-drug resistant Gram-negative pathogens [1-5]. Multi-drug resistance in Pseudomonas aeruginosa, Acinetobacter species, Escherichia coli, and Klebsiella species limits the availability of antimicrobial options in the treatment of these infections [6,7]. Regardless of antimicrobial selection and intervention strategies, clinical outcome of these infections remain undesirable in the form of potential extended length of stay, extended drug exposures leading to adverse effects, or mortality [8-11]. The challenges of this clinical scenario are not only in management of individual infections at the patient level but also in wide-spread antimicrobial use and application at institution and geographical levels to curtail resistance. Antimicrobial stewardship programs are focused to address the need for prudent and optimal use of antibiotics at institution levels [12-14]. These programs have demonstrable impact on selective use of antimicrobials, however, little progress on ameliorating resistance rates are currently observed [15]. During an era where there is a lack of new antimicrobials and antimicrobials with novel mechanisms of action, there is an obvious need for heightened attention to dosing current antibiotics to optimize their efficacy and to curtail resistance development. There has been an increase in pharmacokinetic/pharmacodynamic (PK/PD) investigations of current and old antimicrobials in the past two decades; however, the translation of this knowledge to the bedside is limited by sparse prospective clinical data and the need for more research in specific antimicrobial applications. Clinicians must resort to the collective interpretation of pharmacokinetic properties, in-vitro data, experimental simulations, animal studies, and retrospective evaluations to implement dosing strategies for a variety of infections. It is critical that clinicians optimize therapy both to maximize clinical outcomes and to minimize the risk of resistance. This review will primarily focus on the application of $\mathrm{PK} / \mathrm{PD}$ principles in the treatment and prevention of gram-negative resistance with commonly used antimicrobials.

\section{PK/PD Principles}

The pharmacokinetics/pharmacodynamics of an antimicrobial can be described as the complex relationship between the pharmacokinetic exposure characteristics of an antimicrobial in a human to the effect of that antimicrobial [16,17]. The pharmacokinetic exposure has been typically characterized by steady-state parameters such as peak concentration, the duration that concentrations remain at specific levels, and the area under-the-curve while the antimicrobial effect has been typically characterized by reduction of bacterial colonies in attempts to quantify magnitudes of this relationship. This relationship can be possible when there is an association between drug exposure characteristics to antimicrobial potency such as the minimum inhibitory concentration (MIC). Therefore, antimicrobial dosing and regimens may be designed to achieve exposure targets that are associated with a higher probability of desirable microbiological and clinical outcomes [17].

The three practical PK/PD parameters that have been mainly investigated are the ratio of peak concentration to the MIC (Cmax/ $\mathrm{MIC})$, the time during which the concentration exceeds the MIC as a percentage of the dosing interval $(\% \mathrm{~T}>\mathrm{MIC})$, and the ratio of the 24-hour area under the concentration-time curve to the MIC (AUC/ MIC or AUIC). AUC is the most measured parameter for many antimicrobials as it can be regarded as the entire exposure of a drug over a given time period. Furthermore, most antimicrobials exhibit linear pharmacokinetics where the AUC is directly proportionate to dose, the most easily manipulated component of an antimicrobial regimen. The peak concentration and the concentration over time can independently affect the AUC. Consider the example of a concentration-time profile of a drug where all PK parameters (peak concentration, absorption rate, time) remain constant with the exception of clearance. Decreasing the rate of clearance of a drug over the same time period increases concentrations throughout the period, thereby, increasing the AUC

*Corresponding author: Raymond Cha, Pharm.D, Eugene Applebaum College of Pharmacy and Health Sciences 259 Mack Ave. Detroit, Michigan 48201, USA, Tel: 313-577-5303; E-mail: r.cha@wayne.edu

Received October 09, 2012; Accepted October 29, 2012; Published October 31 2012

Citation: Cha R, Michienzi SM, Hsaiky L (2012) Antimicrobial Pharmacokinetics and Pharmacodynamics in the Treatment of Nosocomial Gram-negative Infections. Adv Pharmacoepidem Drug Safety S1:005. doi:10.4172/2167-1052. S1-005

Copyright: (c) 2012 Cha R, et al. This is an open-access article distributed under the terms of the Creative Commons Attribution License, which permits unrestricted use, distribution, and reproduction in any medium, provided the original author and source are credited. 
(and AUC/MIC) and potentially durations that concentrations remain above a threshold (as in the \% $>\mathrm{MIC}$ ). Thus certain antimicrobials may be linked with both AUC/MIC and another parameter, but one parameter typically prevails with a relatively higher association. Animal and in vitro experimental models have demonstrated that this interdependence can be addressed with dose-fractionated approaches $[16,18]$.

The application of each of these $\mathrm{PK} / \mathrm{PD}$ parameters varies according to the microbial killing characteristics of different antimicrobial drug classes. Antimicrobials that exhibit increased bacterial kill with increasing concentrations are categorized as concentration-dependent drugs. Antimicrobials such as aminoglycosides, fluoroquinolones, and daptomycin exhibit increasing bactericidal activity as concentrations increase with relatively little dependence on duration of exposure. The PK/PD parameters, Cmax/MIC or AUC/MIC ratio, logically correlates with their activity [19-21]. Conversely, antimicrobials that reach maximal killing early in the presence of low concentrations within clinically achievable ranges and with higher dependence on duration of exposure, are categorized as concentration-independent or time-dependent antimicrobials. Antimicrobials such as betalactams, carbapenems, linezolid and tigecycline are considered timedependent drugs whose activity is maximized as the duration that their concentrations remain above a multi-fold of the MIC (T>MIC) is extended [22-24].

Negri et al. was one of the first investigators to demonstrate that the selection of resistant bacteria is associated with antimicrobial concentrations [25]. These investigators hypothesized that there is a selection window of concentrations in which there is a higher potential for selection of strains with increased levels of resistance. This concept soon translated to the 'mutant prevention concentration' (MPC), which describes the lowest antimicrobial concentration that is able to prevent the growth of the least susceptible single-step mutant. Therefore, the mutant selection window describes the concentration range between the MPC and the MIC of the more susceptible sub-population of a given organism [26]. At this point, several experimental models have utilized this pharmacodynamic endpoint and there is a need for further determination of the application of this resistance measure in experimental and clinical evaluations.

\section{Antimicrobial Resistance}

The prevalence of antimicrobial resistance is threatening the human race on a global level. The World Health Organization has labeled this critical issue as one of the three greatest threats to human health [27-29]. There is justified concern over the increased morbidity and mortality in patients with infections due to antimicrobial-resistant gram-positive, gram-negative, fungal, and viral pathogens. The overwhelming prevalence of resistant gram-positive organisms has resulted in the development of several potent and efficacious antimicrobials in the forms of linezolid, daptomycin, tigecycline and ceftaroline to battle infections caused by methicillin-resistant Staphylococcus aureus (MRSA) or vancomycin-resistant Enterococci (VRE) [30-32]. Tigecycline and ceftaroline are broad-spectrum antimicrobials that exhibit activity against gram-negative pathogens as well. However, there is an absence of new antimicrobials with novel mechanisms of action and narrow spectrum of activity against resistant-gram-negative pathogens. This deficiency necessitates heightened assessment of PK/ PD characteristics of ubiquitous antimicrobials that are used both broadly and specifically for nosocomial gram-negative infections: beta-lactams (i.e. piperacillin/tazobactam, cefepime) carbapenems (i.e. meropenem, doripenem) [33].

\section{Beta-lactams}

Beta-lactam-resistant organisms have been reported in the literature as far back as the 1950s following the development of penicillin, as well as prior to its introduction into clinical use [34]. Predictably, the introduction of cephalosporins and other broad-spectrum betalactams was followed by the same timeline of events [35-37]. Of greater concern is the increasing prevalence of extended-spectrum beta-lactamases (ESBL) in Pseudomonas species, Acinetobacter species, and Enterobacteriaceae that render current beta-lactam antimicrobials useless. A worldwide survey in 2004 collected 6156 gram-negative isolates from patients with intra-abdominal infections in 28 different countries. The overall rate of ESBL production was $17 \%$ among $K$ pneumoniae and $10 \%$ among $E$ coli isolates [38]. Similar results were reported by the Tigecycline Evaluation and Surveillance Trial global surveillance study in 2007 [39].

Numerous in vitro and in vivo investigations confirm that $\mathrm{T}>\mathrm{MIC}$ is widely regarded as the main parameter that describes the pharmacodynamic killing activity of beta-lactam antibiotics. The $\% \mathrm{~T}>\mathrm{MIC}$ required for bacteriostasis and bactericidal activity for penicillins and cephalosporins is $30 \%$ and $50 \%$ and $35-40 \%$ and $60-70 \%$, respectively $[16,22,40,41]$. Evidence for $\% \mathrm{~T}>\mathrm{MIC}$ endpoints for betalactams that prevent the development of resistance in Gram-negative organisms is currently sparse. For example, one in vivo experimental study reported that resistant strains of Pseudomonas aeruginosa were only detected in those animals receiving cephalosporins in which the $\% \mathrm{~T}>\mathrm{MIC}$ was less than half of the dosing interval [42]. Until further evidence becomes available, it would be prudent to employ dosing regimens designed at achieving concentrations greater than 4 times the MIC for extended intervals to maximize the likelihood of eradicating entire bacterial populations that may include resistant sub-populations $[43,44]$.

It has been suggested that bacteriostasis and bactericidal-based endpoints may not be adequate to treat serious infections $[45,46]$. Recent retrospective clinical data for critically ill patients suggest higher and longer antimicrobial exposures than those reported in experimental studies [47,48]. McKinnon et al. demonstrated that patients receiving a cephalosporin for serious infections with $\%$ T $>$ MIC of $100 \%$ had significantly better clinical and bacteriological outcomes than patients with $\% T>$ MIC of $<100 \%$. Consequently, maintaining concentrations above the MIC for $90-100 \%$ of the dosing interval with continuous infusion (CI) or extended-infusion (EI) of antimicrobials has been suggested to ensure that minimum PK/PD targets are achieved $[47,49,50]$. Alternatively, time-dependent antimicrobials could be dosed more frequently to achieve higher $\% \mathrm{~T}>\mathrm{MIC}$ but with obvious practical and convenience issues. Targeting a multi-fold of the MIC for extended durations can become difficult to achieve with intermittent dosing of rapidly clearing beta-lactams. This may be especially true when treating critically-ill patients with variable pharmacokinetics and infections due to potential pathogens with higher baseline MIC's or greater resistant sub-populations. Two clinical studies provide evidence for unfavorable outcomes when PK/PD target attainment is not achieved in infections due to pathogens with elevated MIC's. Bhat et al. demonstrated significantly worse 28-day mortality in subjects who had Gram-negative bacteremia with cefepime MICs of $\geq 8 \mu \mathrm{g} / \mathrm{mL}$ vs. those with MICs of $<8 \mu \mathrm{g} / \mathrm{mL}$ (54.8\% vs. $24.1 \%$ ) [51]. In investigations of piperacillin-tazobactam for Pseudomonas aeruginosa bacteremia, Tam et al. demonstrated higher 30-day mortality in subjects with elevated MICs of $32 \mu \mathrm{g} / \mathrm{mL}$ or $64 \mu \mathrm{g} / \mathrm{mL}$ [52].

While CI dosing has been shown to be superior to intermittent dosing 
in in vitro and in vivo experimental studies, numerous comparative clinical studies have demonstrated no significant differences in patient outcome [53-55]. This investigational group of studies is characterized by variety of study design archetypes, heterogeneous subjects, mixed infections, an array of outcome measures, and limited power [50]. An example of a reasonably designed larger comparative clinical study was performed by Lau et al. who investigated CI versus traditional intermittent dosing of piperacillin-tazobactam in 167 patients [56]. They reported cure rates of $86.4 \%$ and $88.4 \%$ for continuous infusion and the intermittent infusion, respectively. Similar microbiological results with no statistical difference was noted in a large variety of clinical trials [5760]. Recent meta-analyses also echo similar outcomes between CI and intermittent dosing of beta lactams across heterogeneous hospitalized patient populations [61-63]. Alternatively, prolonging the infusion time of intermittent dosing regimens has been suggested to enhance the $\% \mathrm{~T}>\mathrm{MIC}$ without some of the $\mathrm{CI}$-associated disadvantages such as drug stability, waste, and toxicity $[48,50]$. Recently, two retrospective clinical trials demonstrated advantages with piperacillin-tazobactam given as extended-infusion (4-hour infusion period) compared to traditional intermittent (30-minute infusion period) dosing regimens in collectively, 288 subjects. Both studies exhibited a statistically significant lower mortality in the EI groups (9.2\% and $12.2 \%)$ versus intermittent dosing groups (17.9\% and 31.6\%) [48,64]. Additionally, a significantly lower length of stay was noted in the single-center cohort study with critically ill patients [48]. In another retrospective investigation, no difference was observed in 30-day mortality rates between patients who received EI piperacillin-tazobactam compared to historical controls who received intermittent infusions [65]. In addition, several publications report successful implementation of hospital wide dosing of extended infusion of piperacillin-tazobactam [66,67].

\section{Carbapenems}

The development of carbapenemases in gram-negative organisms with the ability to hydrolyze carbapenems was foreseeable. Since their introduction to clinical use in the 1980 's, carbapenems have widely been considered as the antimicrobials of choice for the treatment of infections due to resistant-Gram-negative organisms [68-70]. Following historical resistance trends, we now witness an alarming increase of pathogens resistant to carbapenems including outbreaks resulting in overwhelming morbidity and mortality [71-74]. Carbapenem resistance was reported in up to $4.0 \%$ and $10.8 \%$ in Escherichia coli and Klebsiella pneumoniae, respectively, in the National Healthcare Safety Network (NHSN) from isolates collected in 2006-2007 [75]. Surveillance of meropenem susceptibility in the Meropenem Yearly Susceptibility Test Information Collection Program report significant increase in non-susceptible clinical isolates of Klebsiella pneumoniae from $0.6 \%$ in 2004 to $5.6 \%$ in 2008 [76].

The pharmacodynamic activity of carbapenems, possessing the beta-lactam ring in their chemical structure, is best described by timedependent bactericidal activity. Therefore, the PK/PD parameter that is linked with carbapenems is the percent time that the unbound concentration exceeds the MIC (\%T>MIC). Previous experimental in vivo studies have established that carbapenems achieve bacteriostasis and bactericidal maximal activities when $\% \mathrm{~T}>\mathrm{MIC}$ of $20 \%$ and $40 \%$ are achieved, respectively $[16,77,78]$. Relative to beta-lactams, \% $>$ MIC requirements are lower and may be due to their inherent potency and post-antibiotic effect. As discussed earlier for other beta-lactam antibiotics, the same general principles of maximizing the $\% \mathrm{~T}>\mathrm{Max}$ continues to hold true for carbapenems for the same reasons. Thus, conventional dosing schemes may be modified to achieve a target $\% \mathrm{~T}>\mathrm{MIC}$ through extended- or continuous-infusion dosing regimens. A few studies have demonstrated the potential application of CI of carbapenems with comparable outcomes to intermittent dosing in both experimental modeling and human investigations [58,79-82]. For example, a PK/PD study evaluated CI versus intermittent dosing of meropenem at low and high doses [82]. Utilizing Monte Carlo simulation, a population pharmacokinetic modeling technique, it was determined that the probability of target attainment against Pseudomonas aeruginosa was higher with $\mathrm{CI}$ and high dose regimens while no differences were noted between dosing regimens against Klebsiella pneumonia and Enterobacter cloacae. However, the potential utility of the CI dosing strategy is diminished by sparse data and the requirement of frequent intravenous bag exchanges or the use of cold pouches to prevent the rapid drug instability [83].

Given this latter limitation for CI dosing, alternative small and frequent dosing (i.e. $500 \mathrm{mg}$ every 6 hours) of meropenem has been proposed. Several population pharmacokinetic studies utilized the Monte Carlo method to determine the target attainment probability of meropenem given as $500 \mathrm{mg}$ every 6 hours and 1 gm every 8 hours against a variety of Gram-negative pathogens. Pharmacokinetic variables were obtained from healthy volunteers as well as simulated for varying renal function. The probabilities of the two dosing schemes were similar across the simulations $[84,85]$. Several clinical studies evaluating the same meropenem dosing schemes report similar findings with no statistical difference in in-hospital mortality, clinical success, length of stay, or treatment duration [86-88]. Interestingly, secondary pharmacoeconomic evaluations suggests some cost advantages with this alternate dosing. Despite these theoretical advantages, there is concern over the ability to attain adequate $\% \mathrm{~T}>\mathrm{MIC}$ in critically-ill patients with variable pharmacokinetics or who are also infected by pathogens with potentially elevated MICs.

With the beneficial characteristics of slightly longer half-lives and a relatively higher toxicity threshold, carbapenem PK/PD investigations have included the application of EI as well as increasing the dose. Extended-infusions of 3 and 4 hours (as opposed to 30 minute infusions) for meropenem and doripenem, respectively, have been evaluated in multiple experimental model simulations. Li et al developed a meropenem population pharmacokinetic model using patient data with intra-abdominal infections, community-acquired pneumonia, or ventilator-associated pneumonia [89]. Meropenem dosing regimens of $0.5,1$, and 2 grams over 30 minutes and 3 hours every 8 hours were simulated for a fixed susceptibility concentration of $4 \mu \mathrm{g} / \mathrm{mL}$ to represent the standard susceptibility breakpoints for Enterobacteriaceae, Acinetobacter species, and P. aeruginosa. Results indicated that the probability of attaining \% $>$ MIC of $40 \%$ (minimum bactericidal threshold) was increased from 64\% to $90 \%$ when the infusion was extended from 30 minutes to 3 hours for the 1 gram dosing level. The highest attainment probability were observed for simulations of meropenem dosing regimens of 2 g every 8 hours administered as a 3 hour infusion for Acinetobacter species and $P$. aeruginosa. In a comparative population pharmacokinetic study, Lomaestro and Drusano demonstrated that $1 \mathrm{~g}$ of meropenem given every 8 hours as a 3 hour infusion achieved a higher target attainment rate against Pseudomonas aeruginosa than either meropenem at $0.5 \mathrm{~g}$ or imipenem-cilastatin given as $0.5 \mathrm{~g}$ every 6 hours as a 1 hour infusion [90]. Similarly, Jaruratanasirikul et al. evaluated the $\% \mathrm{~T}>\mathrm{MIC}$ attainment in 9 patients with ventilator-associated pneumonia (VAP) and in 8 patients with febrile neutropenia with bacteremia (FNB) who received meropenem consecutively as $1 \mathrm{~g}$ infused over 10 minutes, $1 \mathrm{~g}$ 
infused over 3 hours and $2 \mathrm{~g}$ infused over 3 hours. At an MIC threshold of $4 \mu \mathrm{g} / \mathrm{mL}$, the statistically different mean probabilities of $\% \mathrm{~T}>\mathrm{MIC}$ of $40 \%$ were $57 \%, 73 \%$, and $86 \%$ in VAP and $75.7 \%, 99.24 \%$ and $99.96 \%$ in (FNB), respectively, for the consecutive dosing schemes [91].

Doripenem is a newer carbapenem that shares similar pharmacodynamic and pharmacologic properties as meropenem with the important distinctions that it has an approximately 2 fold lower MIC susceptibility profile, a lower propensity to be hydrolyzed by carbapenemases, and is more stable in intravenous solutions at room temperature [92-94]. In $0.9 \%$ sodium chloride and $5 \%$ dextrose solutions, meropenem is stable for 1 and 4 hours while doripenem is stable for 12 and 4 hours, respectively $[95,96]$. This increased stability potentiates the possibility of administering extended and continuous infusion dosing strategies for doripenem. A population pharmacokinetic study was performed during the early development of doripenem to discern optimal dosing strategies. Monte Carlo simulation revealed that dosing regimens simulated at 4-6 hour infusions produced higher probabilities of target attainment of $\% \mathrm{~T}>\mathrm{MIC}$ of $35 \%$ for organisms with higher MICs [97]. A few population PK/PD studies report similar findings with increased probability of target attainment with prolonged infusion when targeting pathogens with elevated MICs [98-100]. These findings lead to a well-designed large clinical trial evaluating EI dosing of doripenem administered as $500 \mathrm{mg}$ every 8 hours over 4 hours vs. intermittent dosing of imipenem [101]. This was a prospective, randomized trial in 531 subjects with ventilator-associated pneumonia across multiple institutions. Doripenem was found to be noninferior to imipenem in the primary efficacy measures of clinical success in both the clinical modified intent-to-treat analysis (59.0\% vs. $57.8 \%$, respectively) as well as in clinically evaluable subjects $(68.3 \%$ vs. $64.2 \%)$. Additionally, higher cure rates were observed for doripenem in subjects with higher Acute Physiology and Chronic Health Evaluation II scores and older ages.

\section{Overall}

Despite established relation between the pharmacodynamic activity and the clinically-relevant exposures of these antimicrobials, CI or EI dosing of beta-lactam and carbapenem antibiotics remains uncommon. The disparity between the evidence in preclinical experiments and clinical reports, the heterogeneity of study designs, the paucity of data from prospective clinical evaluations, and practical dosing design considerations contributes to the hesitancy of the clinician to shift treatment paradigms away from half a century of traditional dosing of time-dependent antimicrobials.

\section{PK/PD challenges}

Since the first description of the influence of pharmacokinetic exposure to the bactericidal activity of penicillin in the 1950, significant progress has been made to elucidate the role of PK/PD principles in the design and application of antimicrobial therapy for optimizing bacterial eradication [102]. There is also a growing body of literature with compelling evidence for the prevention of resistance emergence with PK/PD target attainment. However, a significant portion of this data comes from investigations in gram-positive organisms and with other antimicrobials such as fluoroquinolones [103]. Little progress has been made to elucidate the application of $\mathrm{PK} / \mathrm{PD}$ principles in the prevention of resistance specifically for beta-lactams and carbapenems, widely accepted antimicrobials of choice for Gram-negative infections [104]. Thus, there is a potential that the PK/PD parameter that reflects resistance suppression may not be the same parameter that is associated with bacterial eradication [105]. In one of these investigations, Tam et al. demonstrated that meropenem dosing that resulted in $\mathrm{Cmin}$ (trough concentrations)/MIC ratios of less than 6.2 resulted in selection of resistant mutations in P. aeruginosa when tested in an in vitro hollow fibre model [106]. Evidence of negative clinical consequences from falling short of PK/PD targets and the difficulty in achieving parameter thresholds in infections due to resistant pathogens, emphasizes the need for research with the pharmacodynamic endpoint of preventing selection of resistance that confer MIC elevations [47,51,52]. This becomes a very challenging task given the necessity to account for a variety of factors. Examples of some of these factors include pharmacokinetic inter- and intra-individual variability, selection of genotypic or phenotypic measures of resistance, selection of the PK/PD parameter that correlates to the desired PD outcome, and application of alternate therapeutic options. Until more progress has been made for resistance prevention strategies in experimental and clinical studies, clinicians are challenged with translating experimental evidence of bacterial eradication to the bedside.

There is a lack of prospective clinical trials that incorporate endpoint measures for the prevention of resistance in general, and specifically for Gram-negative organisms. One exception is a recent prospective clinical trial (mentioned above) that included resistance as a secondary endpoint in the comparative evaluation of the safety and efficacy of extended infusion of doripenem vs. traditional imipenem in patients with ventilator-associated pneumonia. This secondary subgroup analysis reported that $18 \%$ ( 5 of 28) of $P$. aeruginosa isolates had minimum inhibitory concentration $\geq$ or $=8 \mu \mathrm{g} / \mathrm{mL}$ at baseline or following therapy in the doripenem arm compared with 64\% (16 of 25) in the imipenem treatment group. The authors concluded that one of the possibilities for this finding could be improved PK/PD target attainment with the doripenem extended infusion group [101] There is a great need for more prospective clinical trials across different populations and for a variety of infections that utilize established PK/ PD parameters that are associated with the development of resistance. Furthermore, increased clinical evidence of the influence of collective $\mathrm{PK} / \mathrm{PD}$ targets in combination therapies as well as duration of therapies on the prevention of resistance would contribute to the application of antimicrobial treatment strategies.

Inter- and intra-patient pharmacokinetic variability renders the design of dosing regimens difficult when treating patients. The influence of volume of distribution and renal clearance can drastically change the ability of specific dosing regimens to achieve desirable PK/ PD targets. For instance, the rapid volume expansion that occurs early in septic patients can decrease the peak concentration that's achieved with traditional dosing regimens resulting in overall reduced drug exposures and an abbreviated time for concentrations to fall below MICs [107-109]. As these patients develop acute renal dysfunction, the concentration-time profile may shift in favor of time-dependent drugs that have a component of renal clearance. However, these intricate clinical scenarios require a balanced approach of optimizing PK/PD target attainment with avoidance of toxicities. Several Monte Carlo simulations have incorporated varying degrees of renal function to populate their pharmacokinetic variances $[110,111]$. Volume of distribution becomes more complex when considering the clinical significance of reaching sites of infection with active drug. Pharmacokinetic assessments derived from serum concentrations may not necessarily reflect the concentration-time profile of antimicrobials at the site of infection (i.e. lung, bone, cns, skin, etc.). Cmax, absorption into the tissue, and clearance from the tissue can be dramatically different than serum concentration-time parameters [112,113]. Certainly indirect $\mathrm{PK} / \mathrm{PD}$ associations can be made, but this pharmacokinetic 
Citation: Cha R, Michienzi SM, Hsaiky L (2012) Antimicrobial Pharmacokinetics and Pharmacodynamics in the Treatment of Nosocomial Gramnegative Infections. Adv Pharmacoepidem Drug Safety S1:005. doi:10.4172/2167-1052.S1-005

Page 5 of 8

consideration is important in assessments of variability. Additional assessments must be made for the influence of protein binding in the utilization of pharmacokinetic parameters and their variability. It has been suggested that the unbound, free fraction of the drug primarily contributes to its pharmacodynamics activity [114]. Consequently, majority of recent experimental investigations account for this protein binding in their PK/PD simulations. Successful clinical experience with highly-bound drugs ( $>90 \%)$, however, suggests that this factor may be more complex than utilizing a free fraction percentage in modeling and necessitates further elucidation in vivo.

Total of resolution of infection requires both the rapid attainment of PK/PD targets for sufficient duration in order to optimize bacterial eradication and the host's immune defenses. Early PK/PD target attainment not only reduces time to clinical resolution but may also reduce the likelihood of concentrations that may linger at levels that may promote the selection of resistant mutants [115]. Duration of antimicrobials has been demonstrated to have an impact on the selection of resistance in gram-negative organisms. Logically, shortening the course of therapy to the minimum duration required for bacterial eradication may decrease selective pressure. This understanding holds true for the changes that have been observed in overall reduction of utilization of antimicrobials and the subsequent change in antibiogram, as well as in prolonged use of antimicrobials and resistance development [116,117]. The need for additional determination of optimal durations with the balancing act between adequate exposure for clinical resolution and minimum exposure for selective pressure can be highlighted in two investigations. One of the earliest studies investigating this relationship was a prospective clinical trial that discovered the emergence of multidrug resistance in $P$. aeruginosa after 6 days of therapy in subjects with ventilatorassociated pneumonia [118]. More recently, The US Food and Drug Administration notified the public about the early termination of a ventilator-associated pneumonia trial due to safety concerns [119]. In comparison with imipenem/cilastatin, subjects on doripenem $1 \mathrm{gm}$ every 8 hours as extended-infusion (over 4 hours) experienced excess 28 -day all-cause mortality ( 21.5 vs. $14.8 \%$ ) and lower clinical cure rates ( 45.6 vs. $56.8 \%$ ) in the microbiological intent-to-treat analysis. Duration of therapy was fixed at 7 days for the doripenem treatment group and 10 days for the imipenem/cilastatin treatment group. Certainly, there are several other factors beyond duration that could have influenced doripenem's inferiority such as inter- and intra-individual serum pharmacokinetic variability, pharmacokinetics of doripenem in the lung, and clinical factors influencing severity of infection. In attempts to accommodate some of these variables, there has been recent growth of investigations into the utilization of high-dose regimens [120-123].

\section{Summary}

Novel dosing strategies for beta-lactams that optimize pharmacokinetic/pharmacodynamic properties exhibit potential for improved clinical outcomes. Carbapenems exhibit a high probability of attaining its bactericidal and bacteriostatic pharmacodynamic targets with both standard and novel dosing regimens. Pharmacokinetic/ pharmacodynamic profiling is valuable in the design and application of antimicrobial dosing strategies to optimize clinical outcomes. Moreover, the need for expanded experimental and prospective clinical studies that incorporate resistance endpoints is magnified in the face of increasing resistance and decreasing development of novel antibiotics.

\section{References}

1. Vincent JL, Rello J, Marshall J, Silva E, Anzueto A, et al. (2009) International study of the prevalence and outcomes of infection in intensive care units. JAMA 302: 2323-2329.

2. Lockhart SR, Abramson MA, Beekmann SE, Gallagher G, Riedel S, et al (2007) Antimicrobial resistance among Gram-negative bacilli causing infections in intensive care unit patients in the United States between 1993 and 2004. J Clin Microbiol 45: 3352-3359

3. Lu PL, Liu YC, Toh HS, Lee YL, Liu YM, et al. (2012) Epidemiology and antimicrobial susceptibility profiles of Gram-negative bacteria causing urinary tract infections in the Asia-Pacific region: 2009-2010 results from the Study for Monitoring Antimicrobial Resistance Trends (SMART). Int J Antimicrob Agents 40: S37-S43.

4. Kallen AJ, Hidron Al, Patel J, Srinivasan A (2010) Multidrug resistance among gram-negative pathogens that caused healthcare-associated infections reported to the National Healthcare Safety Network, 2006-2008. Infect Control Hosp Epidemiol 31: 528-531.

5. Rosenthal VD, Maki DG, Jamulitrat S, Medeiros EA, Todi SK, et al. (2010) International Nosocomial Infection Control Consortium (INICC) report, data summary for 2003-2008, issued June 2009. Am J Infect Control 38: 95-104.

6. Ho J, Tambyah PA, Paterson DL (2010) Multiresistant Gram-negative infections: a global perspective. Curr Opin Infect Dis 23: 546-553.

7. Obritsch MD, Fish DN, MacLaren R, Jung R (2005) Nosocomial infections due to multidrug-resistant Pseudomonas aeruginosa: epidemiology and treatment options. Pharmacotherapy 25: 1353-1364

8. Qureshi ZA, Paterson DL, Peleg AY, Adams-Haduch JM, Shutt KA, et al. (2012) Clinical characteristics of bacteraemia caused by extended-spectrum ß-lactamase-producing Enterobacteriaceae in the era of CTX-M-type and KPCtype ß-lactamases. Clin Microbiol Infect 18: 887-893.

9. Gudiol C, Calatayud L, Garcia-Vidal C, Lora-Tamayo J, Cisnal M, et al. (2010) Bacteraemia due to extended-spectrum beta-lactamase-producing Escherichia coli (ESBL-EC) in cancer patients: clinical features, risk factors, molecular epidemiology and outcome. J Antimicrob Chemother 65: 333-341.

10. Tam VH, Rogers CA, Chang KT, Weston JS, Caeiro JP, et al. (2010) Impact of multidrug-resistant Pseudomonas aeruginosa bacteremia on patient outcomes. Antimicrob Agents Chemother 54: 3717-3722.

11. Mauldin PD, Salgado CD, Hansen IS, Durup DT, Bosso JA (2010) Attributable hospital cost and length of stay associated with health care-associated infections caused by antibiotic-resistant gram-negative bacteria. Antimicrob Agents Chemother 54: 109-115.

12. Tamma PD, Cosgrove SE (2011) Antimicrobial stewardship. Infect Dis Clin North Am 25: 245-260.

13. Shlaes DM, Gerding DN, John JF Jr, Craig WA, Bornstein DL, et al. (1997) Society for Healthcare Epidemiology of America and Infectious Diseases Society of America Joint Committee on the Prevention of Antimicrobia Resistance: guidelines for the prevention of antimicrobial resistance in hospitals. Clin Infect Dis 25: 584-599.

14. Nowak MA, Nelson RE, Breidenbach JL, Thompson PA, Carson PJ (2012) Clinical and economic outcomes of a prospective antimicrobial stewardship program. Am J Health Syst Pharm 69: 1500-1508.

15. Slain D, Sarwari AR, Petros KO, McKnight RL, Sager RB, et al. (2011) Impact of a Multimodal Antimicrobial Stewardship Program on Pseudomonas aeruginosa Susceptibility and Antimicrobial Use in the Intensive Care Unit Setting. Crit Care Res Pract 2011: 416426.

16. Craig WA (1998) Pharmacokinetic/pharmacodynamic parameters: rationale for antibacterial dosing of mice and men. Clin Infect Dis 26: 1-10.

17. Drusano GL (2007) Pharmacokinetics and pharmacodynamics of antimicrobials. Clin Infect Dis 45: S89-95.

18. Craig WA (2003) Basic pharmacodynamics of antibacterials with clinical applications to the use of beta-lactams, glycopeptides, and linezolid. Infect Dis Clin North Am 17: 479-501.

19. Moore RD, Lietman PS, Smith CR (1987) Clinical response to aminoglycoside therapy: importance of the ratio of peak concentration to minimal inhibitory concentration. J Infect Dis 155: 93-99.

20. Forrest A, Nix DE, Ballow CH, Goss TF, Birmingham MC, et al. (1993) Pharmacodynamics of intravenous ciprofloxacin in seriously ill patients. Antimicrob Agents Chemother 37: 1073-1081. 
Citation: Cha R, Michienzi SM, Hsaiky L (2012) Antimicrobial Pharmacokinetics and Pharmacodynamics in the Treatment of Nosocomial Gramnegative Infections. Adv Pharmacoepidem Drug Safety S1:005. doi:10.4172/2167-1052.S1-005

21. Fenton C, Keating GM, Curran MP (2004) Daptomycin. Drugs 64: 445-455.

22. Craig WA (1995) Interrelationship between pharmacokinetics and pharmacodynamics in determining dosage regimens for broad-spectrum cephalosporins. Diagn Microbiol Infect Dis 22: 89-96.

23. Vogelman B, Gudmundsson S, Leggett J, Turnidge J, Ebert S, et al. (1988) Correlation of antimicrobial pharmacokinetic parameters with therapeutic efficacy in an animal model. J Infect Dis 158: 831-847.

24. Hatano K, Wakai Y, Watanabe Y, Mine Y (1994) Simulation of human plasma levels of beta-lactams in mice by multiple dosing and the relationship between the therapeutic efficacy and pharmacodynamic parameters. Chemotherapy 40 : 1-7.

25. Negri MC, Morosini MI, Loza E, Baquero F (1994) In vitro selective antibiotic concentrations of beta-lactams for penicillin-resistant Streptococcus pneumoniae populations. Antimicrob Agents Chemother 38: 122-125.

26. Drlica K (2003) The mutant selection window and antimicrobial resistance. $J$ Antimicrob Chemother 52: 11-17.

27. Hawkey PM, Jones AM (2009) The changing epidemiology of resistance. J Antimicrob Chemother 64: i3-10.

28. Zhang R, Eggleston K, Rotimi V, Zeckhauser RJ (2006) Antibiotic resistance as a global threat: evidence from China, Kuwait and the United States. Global Health 2: 6.

29. Moland ES, Hanson ND, Black JA, Hossain A, Song W, et al. (2006) Prevalence of newer beta-lactamases in gram-negative clinical isolates collected in the United States from 2001 to 2002. J Clin Microbiol 44: 3318-3324.

30. Welte T, Pletz MW (2010) Antimicrobial treatment of nosocomial meticillinresistant Staphylococcus aureus (MRSA) pneumonia: current and future options. Int J Antimicrob Agents 36: 391-400.

31. Micek ST (2007) Alternatives to vancomycin for the treatment of methicillinresistant Staphylococcus aureus infections. Clin Infect Dis 45: S184-190.

32. Saravolatz LD, Stein GE, Johnson LB (2011) Ceftaroline: a novel cephalosporin with activity against methicillin-resistant Staphylococcus aureus. Clin Infect Dis 52: $1156-1163$

33. Talbot GH, Bradley J, Edwards JE Jr, Gilbert D, Scheld M, et al. (2006) Bad bugs need drugs: an update on the development pipeline from the Antimicrobial Availability Task Force of the Infectious Diseases Society of America. Clin Infect Dis 42: 657-668.

34. EAGLE H (1954) The multiple mechanisms of penicillin resistance. J Bacterio 68: 610-616.

35. Rolinson GN (1991) Evolution of beta-lactamase inhibitors. Rev Infect Dis 13 Suppl 9: S727-732.

36. Jacoby GA, Munoz-Price LS (2005) The new beta-lactamases. N Engl J Med 352: 380-391.

37. Arias CA, Murray BE (2009) Antibiotic-resistant bugs in the 21st century--a clinical super-challenge. N Engl J Med 360: 439-443.

38. Rossi F, Baquero F, Hsueh PR, Paterson DL, Bochicchio GV, et al. (2006) In vitro susceptibilities of aerobic and facultatively anaerobic Gram-negative bacilli isolated from patients with intra-abdominal infections worldwide: 2004 results from SMART (Study for Monitoring Antimicrobial Resistance Trends). J Antimicrob Chemother 58: 205-210.

39. Dowzicky MJ, Park CH (2008) Update on antimicrobial susceptibility rates among gram-negative and gram-positive organisms in the United States: results from the Tigecycline Evaluation and Surveillance Trial (TEST) 2005 to 2007. Clin Ther 30: 2040-2050.

40. Mouton JW, den Hollander JG (1994) Killing of Pseudomonas aeruginosa during continuous and intermittent infusion of ceftazidime in an in vitro pharmacokinetic model. Antimicrob Agents Chemother 38: 931-936.

41. Mouton JW, Punt N, Vinks AA (2007) Concentration-effect relationship of ceftazidime explains why the time above the MIC is 40 percent for a static effect in vivo. Antimicrob Agents Chemother 51: 3449-3451.

42. Fantin B, Farinotti R, Thabaut A, Carbon C (1994) Conditions for the emergence of resistance to cefpirome and ceftazidime in experimental endocarditis due to Pseudomonas aeruginosa. J Antimicrob Chemother 33: 563-569.

43. Odenholt I, Gustafsson I, Lowdin E, Cars O (2003) Suboptimal antibiotic dosage as a risk factor for selection of penicillin-resistant Streptococcus pneumoniae: in vitro kinetic model. Antimicrob Agents Chemother 47: 518-523.

44. Lodise TP Jr, Lomaestro B, Drusano GL (2007) Piperacillin-tazobactam fo Pseudomonas aeruginosa infection: clinical implications of an extendedinfusion dosing strategy. Clin Infect Dis 44: 357-363.

45. Scaglione F, Paraboni L (2008) Pharmacokinetics/pharmacodynamics of antibacterials in the Intensive Care Unit: setting appropriate dosing regimens. Int J Antimicrob Agents 32: 294-301.

46. Adembri C, Novelli A (2009) Pharmacokinetic and pharmacodynamic parameters of antimicrobials: potential for providing dosing regimens that are less vulnerable to resistance. Clin Pharmacokinet 48: 517-528.

47. McKinnon PS, Paladino JA, Schentag JJ (2008) Evaluation of area under the inhibitory curve (AUIC) and time above the minimum inhibitory concentration ( $\mathrm{T}>\mathrm{MIC}$ ) as predictors of outcome for cefepime and ceftazidime in serious bacterial infections. Int J Antimicrob Agents 31: 345-351.

48. Lodise TP Jr, Lomaestro B, Drusano GL (2007) Piperacillin-tazobactam fo Pseudomonas aeruginosa infection: clinical implications of an extendedinfusion dosing strategy. Clin Infect Dis 44: 357-363.

49. Sinnollareddy MG, Roberts MS, Lipman J, Roberts JA (2012) B-Lactam pharmacokinetics and pharmacodynamics in critically ill patients and strategies for dose optimization: a structured review. Clin Exp Pharmacol Physiol 39: 489496.

50. Abdul-Aziz MH, Dulhunty JM, Bellomo R, Lipman J, Roberts JA (2012) Continuous beta-lactam infusion in critically ill patients: the clinical evidence. Ann Intensive Care 2: 37

51. Bhat SV, Peleg AY, Lodise TP Jr, Shutt KA, Capitano B, et al. (2007) Failure of current cefepime breakpoints to predict clinical outcomes of bacteremia caused by gram-negative organisms. Antimicrob Agents Chemother 51: 4390-4395.

52. Tam VH, Gamez EA, Weston JS, Gerard LN, Larocco MT, et al. (2008) Outcomes of bacteremia due to Pseudomonas aeruginosa with reduced susceptibility to piperacillin-tazobactam: implications on the appropriateness of the resistance breakpoint. Clin Infect Dis 46: 862-867.

53. Alou L, Aguilar L, Sevillano D, Gimenez MJ, Echeverria O, et al. (2005) Is there a pharmacodynamic need for the use of continuous versus intermittent infusion with ceftazidime against Pseudomonas aeruginosa? An in vitro pharmacodynamic model. J Antimicrob Chemother 55: 209-213.

54. Roosendaal R, Bakker-Woudenberg IA, van den Berg JC, Michel MF (1985) Therapeutic efficacy of continuous versus intermittent administration of ceftazidime in an experimental Klebsiella pneumoniae pneumonia in rats. $J$ Infect Dis 152: 373-378.

55. Gerber AU, Craig WA, Brugger HP, Feller C, Vastola AP, et al. (1983) Impact of dosing intervals on activity of gentamicin and ticarcillin against Pseudomonas aeruginosa in granulocytopenic mice. J Infect Dis 147: 910-917.

56. Lau WK, Mercer D, Itani KM, Nicolau DP, Kuti JL, et al. (2006) Randomized, open-label, comparative study of piperacillin-tazobactam administered by continuous infusion versus intermittent infusion for treatment of hospitalized patients with complicated intra-abdominal infection. Antimicrob Agents Chemother 50: 3556-3561

57. Roberts JA, Boots R, Rickard CM, Thomas P, Quinn J, et al (2007) continuous infusion ceftriaxone better than once-a-day dosing in intensive care? A randomized controlled pilot study. J Antimicrob Chemother 59: 285291

58. Sakka SG, Glauner AK, Bulitta JB, Kinzig-Schippers M, Pfister W, et al. (2007) Population pharmacokinetics and pharmacodynamics of continuous versus short-term infusion of imipenem-cilastatin in critically ill patients in a randomized, controlled trial. Antimicrob Agents Chemother 51: 3304-3310.

59. Bodey GP, Ketchel SJ, Rodriguez V (1979) A randomized study of carbenicillin plus cefamandole or tobramycin in the treatment of febrile episodes in cancer patients. Am J Med 67: 608-616.

60. Rafati MR, Rouini MR, Mojtahedzadeh M, Najafi A, Tavakoli H, et al. (2006) Clinical efficacy of continuous infusion of piperacillin compared with intermittent dosing in septic critically ill patients. Int J Antimicrob Agents 28: 122-127.

61. Roberts JA, Webb S, Paterson D, Ho KM, Lipman J (2009) A systematic review on clinical benefits of continuous administration of beta-lactam antibiotics. Crit Care Med 37: 2071-2078. 
Citation: Cha R, Michienzi SM, Hsaiky L (2012) Antimicrobial Pharmacokinetics and Pharmacodynamics in the Treatment of Nosocomial Gramnegative Infections. Adv Pharmacoepidem Drug Safety S1:005. doi:10.4172/2167-1052.S1-005

62. Kasiakou SK, Sermaides GJ, Michalopoulos A, Soteriades ES, Falagas ME (2005) Continuous versus intermittent intravenous administration of antibiotics: a meta-analysis of randomised controlled trials. Lancet Infect Dis 5: 581-589.

63. Tamma PD, Putcha N, Suh YD, Van Arendonk KJ, Rinke ML (2011) Does prolonged $\beta$-lactam infusions improve clinical outcomes compared to intermittent infusions? A meta-analysis and systematic review of randomized, controlled trials. BMC Infect Dis 11: 181

64. Yost RJ, Cappelletty DM (2011) The Retrospective Cohort of ExtendedInfusion Piperacillin-Tazobactam (RECEIPT) study: a multicenter study. Pharmacotherapy 31: 767-775.

65. Patel GW, Patel N, Lat A, Trombley K, Enbawe S, et al. (2009) Outcomes of extended infusion piperacillin/tazobactam for documented Gram-negative infections. Diagn Microbiol Infect Dis 64: 236-240.

66. Xamplas RC, Itokazu GS, Glowacki RC, Grasso AE, Caquelin C, et al. (2010) Implementation of an extended-infusion piperacillin-tazobactam program at an urban teaching hospital. Am J Health Syst Pharm 67: 622-628.

67. Nichols KR, Knoderer CA, Cox EG, Kays MB (2012) System-wide implementation of the use of an extended-infusion piperacillin/tazobactam dosing strategy: feasibility of utilization from a children's hospital perspective. Clin Ther 34: 1459-1465.

68. Bradley JS, Garau J, Lode H, Rolston KV, Wilson SE, et al. (1999) Carbapenems in clinical practice: a guide to their use in serious infection. Int $J$ Antimicrob Agents 11: 93-100

69. Paterson DL (2000) Recommendation for treatment of severe infections caused by Enterobacteriaceae producing extended-spectrum beta-lactamases (ESBLs). Clin Microbiol Infect 6: 460-463.

70. Papp-Wallace KM, Endimiani A, Taracila MA, Bonomo RA (2011) Carbapenems: past, present, and future. Antimicrob Agents Chemother 55: 4943-4960.

71. Nordmann P, Picazo JJ, Mutters R, Korten V, Quintana A, et al. (2011) Comparative activity of carbapenem testing: the COMPACT study. J Antimicrob Chemother 66: 1070-1078.

72. Bratu S, Landman D, Haag R, Recco R, Eramo A, et al. (2005) Rapid spread of carbapenem-resistant Klebsiella pneumoniae in New York City: a new threat to our antibiotic armamentarium. Arch Intern Med 165: 1430-1435.

73. Mena A, Plasencia V, Garcia L, Hidalgo O, Ayestaran Jl, et al. (2006) Characterization of a large outbreak by CTX-M-1-producing Klebsiella pneumoniae and mechanisms leading to in vivo carbapenem resistance development. J Clin Microbiol 44: 2831-2837.

74. Schwaber MJ, Klarfeld-Lidji S, Navon-Venezia S, Schwartz D, Leavitt A, et al. (2008) Predictors of carbapenem-resistant Klebsiella pneumoniae acquisition among hospitalized adults and effect of acquisition on mortality. Antimicrob Agents Chemother 52: 1028-1033.

75. Hidron AI, Edwards JR, Patel J, Horan TC, Sievert DM, et al. (2008) Nationa Healthcare Safety Network Team; Participating National Healthcare Safety Network Facilities. NHSN annual update: Antimicrobial-resistant pathogens associated with healthcare-associated infections: Annual summary of data reported to the National Healthcare Safety Network at the Centers for Disease Control and Prevention, 2006-2007. Infect Control Hosp Epidemiol 29: 9961011.

76. Rhomberg PR, Jones RN (2009) Summary trends for the Meropenem Yearly Susceptibility Test Information Collection Program: a 10-year experience in the United States (1999-2008). Diagn Microbiol Infect Dis 65: 414-426.

77. Craig WA (1997) The pharmacology of meropenem, a new carbapenem antibiotic. Clin Infect Dis 24: S266-275.

78. Ong CT, Tessier PR, Li C, Nightingale CH, Nicolau DP (2007) Comparative in vivo efficacy of meropenem, imipenem, and cefepime against Pseudomonas aeruginosa expressing MexA-MexB-OprM efflux pumps. Diagn Microbiol Infect Dis 57: 153-161.

79. Thalhammer F, TraunmÃ $1 / 4$ ller F, El Menyawi I, Frass M, Hollenstein UM, et al. (1999) Continuous infusion versus intermittent administration of meropenem in critically ill patients. J Antimicrob Chemother 43: 523-527.

80. Roberts JA, Kirkpatrick CM, Roberts MS, Robertson TA, Dalley AJ, et al. (2009) Meropenem dosing in critically ill patients with sepsis and without renal dysfunction: intermittent bolus versus continuous administration? Monte Carlo dosing simulations and subcutaneous tissue distribution. J Antimicrob Chemother 64: 142-150.
81. Lorente L, Lorenzo L, Martin MM, Jimenez A, Mora ML (2006) Meropenem by continuous versus intermittent infusion in ventilator-associated pneumonia due to gram-negative bacilli. Ann Pharmacother 40: 219-223

82. Krueger WA, Bulitta J, Kinzig-Schippers M, Landersdorfer C, Holzgrabe U, et al. (2005) Evaluation by monte carlo simulation of the pharmacokinetics of two doses of meropenem administered intermittently or as a continuous infusion in healthy volunteers. Antimicrob Agents Chemother 49: 1881-1889.

83. Mattoes HM, Kuti JL, Drusano GL, Nicolau DP (2004) Optimizing antimicrobia pharmacodynamics: dosage strategies for meropenem. Clin Ther 26: 11871198.

84. Kotapati S, Nicolau DP, Nightingale CH, Kuti JL (2004) Clinical and economic benefits of a meropenem dosage strategy based on pharmacodynamic concepts. Am J Health Syst Pharm 61: 1264-1270.

85. Kuti JL, Florea NR, Nightingale CH, Nicolau DP (2004) Pharmacodynamics of meropenem and imipenem against Enterobacteriaceae, Acinetobacter baumannii, and Pseudomonas aeruginosa. Pharmacotherapy 24: 8-15.

86. Ariano RE, Nyhlén A, Donnelly JP, Sitar DS, Harding GK, et al. (2005) Pharmacokinetics and pharmacodynamics of meropenem in febrile neutropenic patients with bacteremia. Ann Pharmacother 39: 32-38

87. Arnold HM, McKinnon PS, Augustin KM, Hladnik LM, Casabar E, et al. (2009) Assessment of an alternative meropenem dosing strategy compared with imipenem-cilastatin or traditional meropenem dosing after cefepime failure or intolerance in adults with neutropenic fever. Pharmacotherapy 29: 914-923.

88. Patel GW, Duquaine SM, McKinnon PS (2007) Clinical outcomes and cost minimization with an alternative dosing regimen for meropenem in a community hospital. Pharmacotherapy 27: 1637-1643.

89. Li C, Kuti JL, Nightingale CH, Nicolau DP (2006) Population pharmacokinetic analysis and dosing regimen optimization of meropenem in adult patients. $J$ Clin Pharmacol 46: 1171-1178.

90. Lomaestro BM, Drusano GL (2005) Pharmacodynamic evaluation of extending the administration time of meropenem using a Monte Carlo simulation. Antimicrob Agents Chemother 49: 461-463.

91. Jaruratanasirikul S, Sriwiriyajan S, Punyo J (2005) Comparison of the pharmacodynamics of meropenem in patients with ventilator-associated pneumonia following administration by 3-hour infusion or bolus injection. Antimicrob Agents Chemother 49: 1337-1339.

92. Keam SJ (2008) Doripenem: a review of its use in the treatment of bacterial infections. Drugs 68: 2021-2057.

93. Mandell L (2009) Doripenem: a new carbapenem in the treatment of nosocomial infection. Clin Infect Dis 49: S1-3.

94. Queenan AM, Shang W, Flamm R, Bush K (2010) Hydrolysis and inhibition profiles of beta-lactamases from molecular classes $A$ to $D$ with doripenem imipenem, and meropenem. Antimicrob Agents Chemother 54: 565-569.

95. Psathas PA, Kuzmission A, Ikeda K, Yasuo S (2008) Stability of doripenem in vitro in representative infusion solutions and infusion bags. Clin Ther 30 2075-2087.

96. Kuti JL, Nightingale CH, Knauft RF, Nicolau DP (2004) Pharmacokinetic properties and stability of continuous-infusion meropenem in adults with cystic fibrosis. Clin Ther 26: 493-501.

97. Bhavnani SM, Hammel JP, Cirincione BB, Wikler MA, Ambrose PG (2005) Use of pharmacokinetic-pharmacodynamic target attainment analyses to support phase 2 and 3 dosing strategies for doripenem. Antimicrob Agents Chemother 49: 3944-3947.

98. Ikawa K, Morikawa N, Ikeda K, Ohge H, Sueda T (2008) Pharmacodynamic assessment of doripenem in peritoneal fluid against Gram-negative organisms: use of population pharmacokinetic modeling and Monte Carlo simulation. Diagn Microbiol Infect Dis 62: 292-297.

99. Samtani MN, Flamm R, Kaniga K, Nandy P (2010) Pharmacokineticpharmacodynamic-model-guided doripenem dosing in critically ill patients. Antimicrob Agents Chemother 54: 2360-2364.

100. Van Wart SA, Andes DR, Ambrose PG, Bhavnani SM (2009) Pharmacokinetic pharmacodynamic modeling to support doripenem dose regimen optimization for critically ill patients. Diagn Microbiol Infect Dis 63: 409-414.

101. Chastre J, Wunderink R, Prokocimer P, Lee M, Kaniga K, et al. (2008) Efficacy and safety of intravenous infusion of doripenem versus imipenem in ventilator- 
Citation: Cha R, Michienzi SM, Hsaiky L (2012) Antimicrobial Pharmacokinetics and Pharmacodynamics in the Treatment of Nosocomial Gramnegative Infections. Adv Pharmacoepidem Drug Safety S1:005. doi:10.4172/2167-1052.S1-005

associated pneumonia: a multicenter, randomized study. Crit Care Med 36: 1089-1096.

102. EAGLE H, FLEISCHMAN R, MUSSELMAN AD (1950) Effect of schedule of administration on the therapeutic efficacy of penicillin; importance of the aggregate time penicillin remains at effectively bactericidal levels. Am J Med 9: 280-299.

103. Mouton JW, Ambrose PG, Canton R, Drusano GL, Harbarth S, et al. (2011) Conserving antibiotics for the future: new ways to use old and new drugs from a pharmacokinetic and pharmacodynamic perspective. Drug Resist Updat 14: $107-117$.

104.Adembri C, Novelli A (2009) Pharmacokinetic and pharmacodynamic parameters of antimicrobials: potential for providing dosing regimens that are less vulnerable to resistance. Clin Pharmacokinet 48: 517-528.

105. Firsov AA, Gilbert D, Greer K, Portnoy YA, Zinner SH (2012) Comparative pharmacodynamics and antimutant potentials of doripenem and imipenem with ciprofloxacin-resistant Pseudomonas aeruginosa in an in vitro model. Antimicrob Agents Chemother 56: 1223-1228.

106. Tam VH, Schilling AN, Neshat S, Poole K, Melnick DA, et al. (2005) Optimization of meropenem minimum concentration/MIC ratio to suppress in vitro resistance of Pseudomonas aeruginosa. Antimicrob Agents Chemother 49: 4920-4927.

107. McKindley DS, Boucher BA, Hess MM, Croce MA, Fabian TC (1996) Pharmacokinetics of aztreonam and imipenem in critically ill patients with pneumonia. Pharmacotherapy 16: 924-931.

108. Pea F, Viale P, Furlanut M (2005) Antimicrobial therapy in critically ill patients: a review of pathophysiological conditions responsible for altered disposition and pharmacokinetic variability. Clin Pharmacokinet 44: 1009-1034.

109. Roberts DM, Roberts JA, Roberts MS, Liu X, Nair P, et al. (2012) Variability of antibiotic concentrations in critically ill patients receiving continuous renal replacement therapy: a multicentre pharmacokinetic study. Crit Care Med 40: 1523-1528.

110. Patel N, Cardone K, Grabe DW, Meola S, Hoy C, et al. (2011) Use of pharmacokinetic and pharmacodynamic principles to determine optimal administration of daptomycin in patients receiving standardized thrice-weekly hemodialysis. Antimicrob Agents Chemother 55: 1677-1683.

111. Koomanachai P, Bulik CC, Kuti JL, Nicolau DP (2010) Pharmacodynamic modeling of intravenous antibiotics against gram-negative bacteria collected in the United States. Clin Ther 32: 766-779.

112. Conte JE Jr, Golden JA, Kelley MG, Zurlinden E (2005) Intrapulmonary pharmacokinetics and pharmacodynamics of meropenem. Int $\mathrm{J}$ Antimicrob Agents 26: 449-456

113. Chandorkar G, Huntington JA, Gotfried MH, Rodvold KA, Umeh O (2012) Intrapulmonary penetration of ceftolozane/tazobactam and piperacillin/ tazobactam in healthy adult subjects. J Antimicrob Chemother 67: 2463-2469.

114. Zeitlinger MA, Derendorf H, Mouton JW, Cars O, Craig WA, et al. (2011) Protein binding: do we ever learn? Antimicrob Agents Chemother 55: 3067 3074.

115. Martinez MN, Papich MG, Drusano GL (2012) Dosing regimen matters: the importance of early intervention and rapid attainment of the pharmacokinetic/ pharmacodynamic target. Antimicrob Agents Chemother 56: 2795-2805.

116. Chastre J, Wolff M, Fagon JY, Chevret S, Thomas F, et al. (2003) Comparison of 8 vs 15 days of antibiotic therapy for ventilator-associated pneumonia in adults: a randomized trial. JAMA 290: 2588-2598.

117. Rice LB (2008) The Maxwell Finland Lecture: for the duration-rational antibiotic administration in an era of antimicrobial resistance and clostridium difficile. Clin Infect Dis 46: 491-496.

118. Dennesen PJ, van der Ven AJ, Kessels AG, Ramsay G, Bonten MJ (2001) Resolution of infectious parameters after antimicrobial therapy in patients with ventilator-associated pneumonia. Am J Respir Crit Care Med 163: 1371-1375

119. US Food and Drug Administration. FDA statement on recently terminated clinical trial with Doribax (doripenem).

120. Jaruratanasirikul S, Wongpoowarak W, Kositpantawong N, Aeinlang N Jullangkoon M (2012) Pharmacodynamics of doripenem in critically ill patients with ventilator-associated Gram-negative bacilli pneumonia. Int J Antimicrob Agents 40: 434-439.

121. Bulik CC, Christensen H, Li P, Sutherland CA, Nicolau DP, et al. (2010) Comparison of the activity of a human simulated, high-dose, prolonged infusion of meropenem against Klebsiella pneumoniae producing the KPC carbapenemase versus that against Pseudomonas aeruginosa in an in vitro pharmacodynamic model. Antimicrob Agents Chemother 54: 804-810.

122. Koomanachai P, Bulik CC, Kuti JL, Nicolau DP (2010) Pharmacodynamic modeling of intravenous antibiotics against gram-negative bacteria collected in the United States. Clin Ther 32: 766-779.

123. Moriyama B, Henning SA, Childs R, Holland SM, Anderson VL, et al. (2010) High-dose continuous infusion beta-lactam antibiotics for the treatment of resistant Pseudomonas aeruginosa infections in immunocompromised patients. Ann Pharmacother 44: 929-935. 